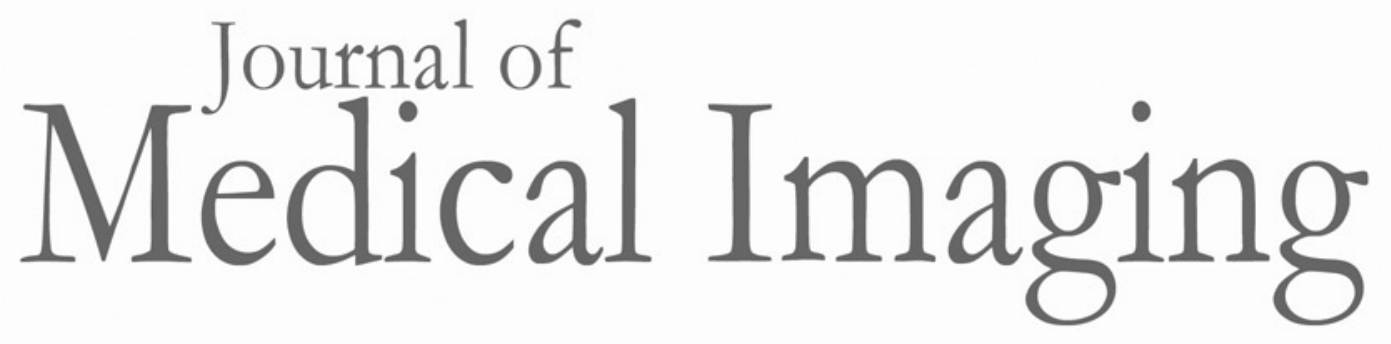

Medicallmaging.SPIEDigitalLibrary.org

\title{
The Spectrum of Medical Imaging in the 2015 International Year of Light
}

Maryellen L. Giger

Martin P. Tornai

\section{SPIE.}



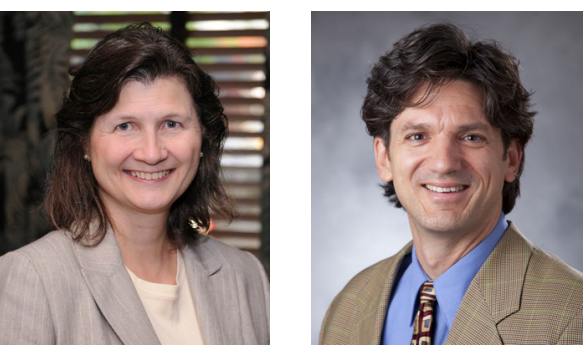

\section{The Spectrum of Medical Imaging in the 2015 International Year of Light}

With this issue of the Journal of Medical Imaging (JMI), I invite you to peruse our cover image (reproduced as Fig. 1), which celebrates the 2015 International Year of Light. I would like to thank Martin Tornai, PhD, of Duke University who graciously gave his time and efforts to design the cover and cowrite this editorial.

In recognition of this year's "International Year of Light," we as medical imaging scientists and engineers contribute to the quest for the improved human condition by providing imaging that spans the entire usable electromagnetic spectrum as we currently know it. From the humblest of beginnings with simple magnification of objects and focusing with lenses, to the biological observation of cells with rudimentary microscopes in the visible spectrum, medical imaging today has both extended away from the visible spectrum towards longer and shorter wavelengths (lower and higher energies) and exploited the hyperspectral nature of the visible, which was once considered "commonplace" or rudimentary. Considering a different perspective of the various imaging modalities across the spectrum, the medically useful information gained spans the anatomic, physiologic, and molecular regimes. Imaging systems and algorithms have been developed as single-modality acquisition systems, and also as multiple modalities with conjoint reconstruction, each informing the other in the quest to optimize image quality and information content.

In addition, the role of light-based technologies in enabling advanced computations in image reconstructions, computer vision and computer-aided diagnosis, machine learning, and 3-D visualizations, as well as in data-enriched storage of medical images, has further extended the potential knowledge "seen" through imaging.

We have much to be proud of as imaging scientists and engineers, recognizing that the breadth of the electromagnetic spectrum offers limitless opportunities for improvement and inquiry, in our quest to answer human life's difficult questions.

This JMI issue's cover montage is but a small tribute to the multitude of medical imaging scientists who have contributed to the wealth of new knowledge as we interrogate the human condition. The grayscale whole body images (from left to right) are acquired from longer wavelength modalities to shorter wavelength modalities spanning the (currently) usable electromagnetic spectrum. The background images include: a coronal magnetic resonance image (MRI) T2 weighted

(C) 2015 Society of Photo-Optical Instrumentation Engineers (SPIE) image slice, a posterior surface infrared (IR) heat map, a coronal noncontrast computed tomography (CT) whole body slice, and a coronal ${ }^{18} \mathrm{~F}$-fluoro-deoxyglucose positron emission tomography (FDG-PET) slice.

The selection of color inset images includes (from top-tobottom, then left to right):

1. Diffusion tensor MRI used to image long white matter tracts within and around the human brain illustrating neuronal connectivity (courtesy of Arthur Toga, PhD).

2. Registered and superimposed coronal proton-MRI (gray scale) and hyperpolarized ${ }^{129} \mathrm{Xe}$ ventilation MRI scan (color scale) within the same patient's lungs, prior to their receiving a bronchial stent (courtesy of Bastiaan Driehuys, PhD).

3. Coronal slice through a microwave-based image reconstruction of the Debye parameter $\varepsilon$ in a boundary outlined heterogeneously dense human breast phantom (courtesy of Susan Hagness, PhD).

4. Juxtaposed optical image of the retina with vertical and horizontal optical coherence tomograph (OCT) slices showing pigmentosa retinopathy (courtesy of Jean-Michel Muratet, MD).

5. Functional 3-D photoacoustic imaging of melanoma (gray scale) in vivo, surrounded by highly optically absorptive blood vessels (courtesy of Lihong Wang).

6. Optical micrograph of HeLa cells stained for microtubules (blue) and actin (red); HeLa cells are the world's first stem cell line, originally derived from the aggressive cervical cancer cells of Henrietta Lacks (courtesy Tom Deerinck/NCMIR).

7. Visualization of the pelvis highlighting the colon, which is imaged with contrast $\mathrm{x}$-ray CT, and after digital surface rendering can be used in virtual colonoscopy, thus avoiding a physical colonoscopy (adapted from http://www.prescan.co.uk).

8. Registered and fused sagittal ${ }^{99 m}$ Tc-sestamibi dedicated molecular SPECT (heat scale) and low-dose CT slice (gray scale) of a patient's uncompressed pendant breast containing two surgically confirmed DCIS loci in the posterior breast (courtesy of Martin Tornai, PhD).

9. Classic, early x-radiograph of Wilhelm Röntgen's wife's hand (adapted from http://en.wikipedia.org/ wiki/100_Photographs_that_Changed_the_World).

10. Transverse pelvic CT slice (gray scale) with multiple superimposed simulated radiotherapy beams, and their cumulative deposited dose isocontours (color scale) at the focus of a tumor (courtesy of Paul Read, MD, PhD).

11. False-colored scanning-electron micrograph of HIV particles (yellow) infecting a human H9 T-cell (turquoise) (adapted from http://biosingularity.com/ category/infection/).

12. Transverse ${ }^{18} \mathrm{~F}$-dopa PET slice through an earlyonset Parkinson patient's brain showing decreased 


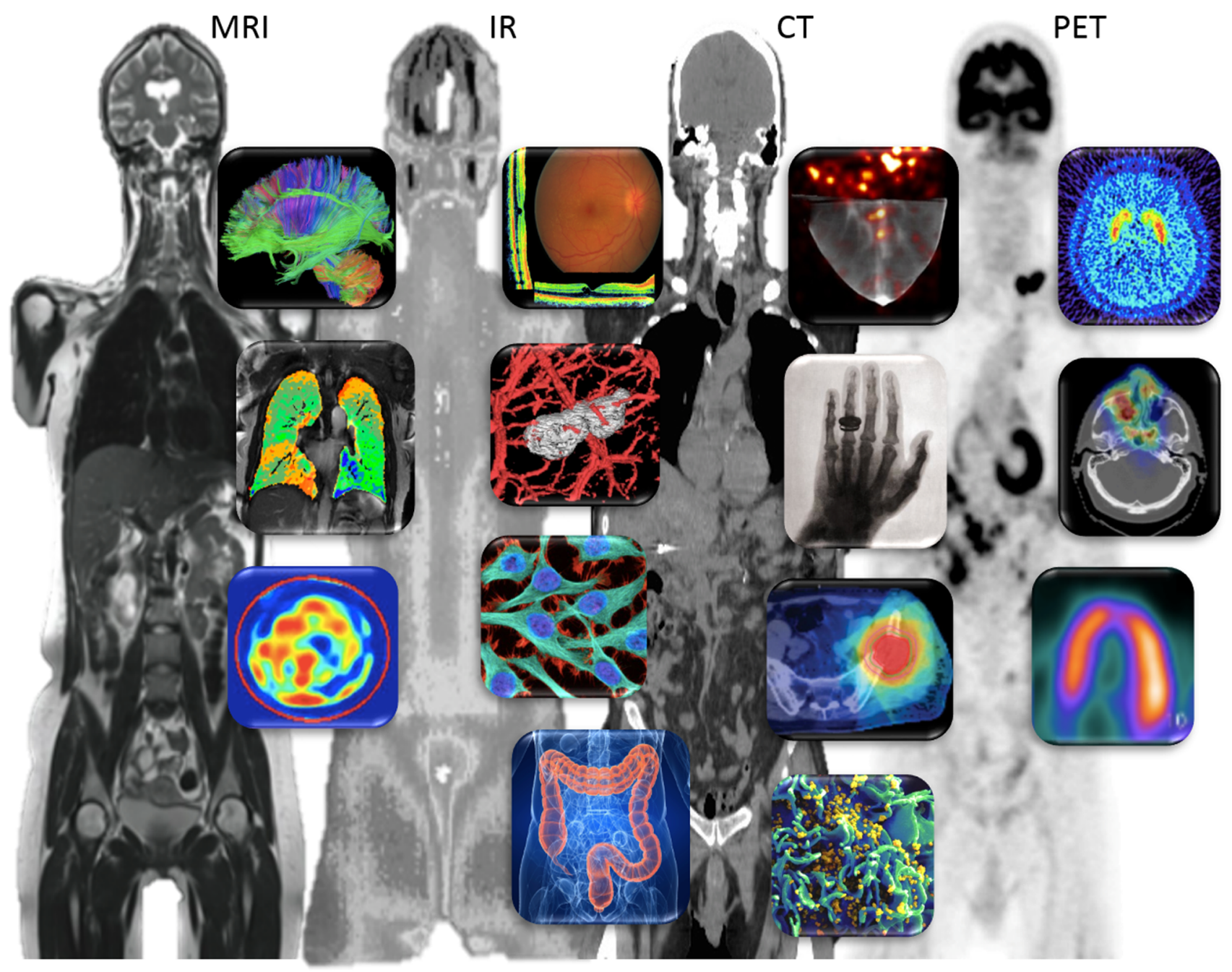

Fig. 1 Journal of Medical Imaging cover image, Vol. 2, No. 3 (2015).

in vivo quantitative uptake in the right putamen (courtesy of Christiaan Schiepers, MD, PhD).

13. Registered and fused transverse head CT (gray scale) and registered superimposed PET image (color) of a patient immediately after therapeutic proton irradiation showing endogenously created ${ }^{15} \mathrm{O}$-positron emitter ( 2 min half-life) within the nasopharyngeal tumor focus (courtesy of Kira Grogg, PhD).

14. Transverse (long axis) cardiac ${ }^{99 m}$ Tc-sestamibi SPECT slice illustrating thinned myocardial apical wall in a rest-stress procedure performed thousands of times daily around the world (adapted from www.medscape.com).

These images are but a small sampling of the breadth of the spectrum of light that is currently being investigated the world over by passionate scientists and engineers on a quest for the betterment of the human condition. We graciously thank all contributors for these images used herein.

We invite you to learn more about the 2015 International Year of Light at www.light2015.org, which notes that the IYL "is a global initiative which will highlight to the citizens of the world the importance of light and optical technologies in their lives, for their futures, and for the development of society." Through this initiative, the United Nations aims to raise global awareness of the vital role of light in medicine, communication, and other disciplines.
Maryellen L. Giger Editor-in-Chief

Martin P. Tornai Associate Editor 